\title{
Electron spin resonance (ESR) detection of irradiated fish containing bone (gilthead sea bream, cod, and swordfish)
}

\author{
A. E. Chiaravalle • M. Mangiacotti • G. Marchesani • \\ G. Vegliante
}

Published online: 13 May 2010

(C) Springer Science+Business Media B.V. 2010

\begin{abstract}
Food irradiation is a preservation method that has been approved by the European Union for a limited number of foods or food products and within a limited range of doses. European Community legislation states that all foods or food ingredients treated in this manner and authorized for sale in the European Union must be clearly labeled, and that market-level inspections must be carried out to ensure compliance with this regulation. To improve detection of irradiated foods, we evaluated the efficacy of electron spin resonance (ESR) spectroscopy for the identification of seafood that had been subjected to irradiation. Three fish species were tested: gilthead sea bream, cod, and swordfish. For each species, 18 samples of flesh-containing bone were analyzed after either irradiation at $0.5,1$, or $3 \mathrm{kGy}$ or no irradiation (control). Induced radicals in irradiated samples produced distinct ESR signals that allowed differentiation from non-irradiated samples. Within the dose range tested, a linear dose-response relationship was observed $\left(R^{2}>0.94\right)$. The gilthead sea bream displayed the highest radiosensitivity. The analytical procedure was validated and successfully used for the routine monitoring of locally marketed seafood. The main aims of the study were to develop a reliable tool for the enforcement of the proper labeling of irradiated foods and to obtain preliminary data on compliance with current laws in the Italian market.
\end{abstract}

Keywords Irradiated foods · Electron spin resonance spectroscopy $\cdot$ Fish products

\author{
Abbreviations \\ ESR electron spin resonance
}

A. E. Chiaravalle $\cdot$ M. Mangiacotti $\cdot$ G. Marchesani $\cdot$ G. Vegliante

Centro di Referenza Nazionale per la Ricerca della Radioattività nel Settore Zootecnico-Veterinario, Istituto Zooprofilattico Sperimentale della Puglia e della Basilicata, Foggia, Italy

A. E. Chiaravalle $(\bowtie)$

Dipartimento di Chimica, Istituto Zooprofilattico Sperimentale della Puglia e della Basilicata, Via Manfredonia 20, 71100 Foggia, Italy

e-mail: e.chiaravalle@izsfg.it 


\section{Introduction}

Today's food industry is faced with several important challenges, including food product deterioration and the constant increase of diseases related to the presence of pathogenic micro-organisms in food products. Thus, adequate and effective food preservation strategies are even more important. Food irradiation is a sterilization process that can improve the microbiological quality of foodstuffs and extend the period in which it can be safely consumed. Due to its numerous positive effects, including those of a commercial nature, this technology has assumed a highly important role in the field of food preservation, and increasingly large numbers of foodstuffs are subjected to this treatment each year. For some time now, countries equipped with adequate facilities for food irradiation have used this technology at well defined doses for the preservation of seafood. Because of the divergent opinions expressed by the main international organizations, the European Union has issued two directives (1999/2/CE and 1999/3/CE), which have been implemented in Italy by Legislative Decree No. 94 of 30 January 2001. With the open market, Italy is obliged to accommodate the presence in its internal market of irradiated fish products treated in other EU states or in extra-European countries. To further safeguard the consumer, the EU legislation provides for official annual checks at the product marketing stage, with the purpose of identifying improperly labeled or unauthorized products. Thus far, trout is the only fish species that has been subjected to interlaboratory validation by electron spin resonance (ESR) spectroscopy for detection of radiation treatment. To meet the specific requirements of the law and to increase acceptance of this type of food preservation technology, we have also validated the UNI EN 1786 method on gilthead sea bream, cod, and sword fish, all of which are widely consumed and likely to be treated with irradiation. We also present the results of a preliminary study on fish products collected from local markets.

\section{Materials and methods}

A total of 54 fresh fish were purchased from local retailers for use in this study: 18 cods, 18 gilthead sea breams, and 18 swordfish). The experimental protocol for identifying irradiated fish products involved two main phases: sample preparation and verification of the method's applicability to the test species. In the first phase, which involved separating the bones from the flesh, bone samples were reduced to fragments and/or powder with an electric grinder and sifted to select fragments with diameters in the range of $0.5-1.0 \mathrm{~mm}$. Fragments were then transferred into Petri dishes measuring $5 \mathrm{~cm}$ in diameter and dried for approximately $3 \mathrm{~h}$ inside a ventilated oven at a temperature of $40 \pm 5^{\circ} \mathrm{C}$. For each species tested, the bone fragments from all 18 specimens were pooled and divided into four aliquots. Three aliquots were irradiated with a Co-60 at $0.5 \mathrm{kGy}, 1.0 \mathrm{kGy}$, or $3.0 \mathrm{kGy}$. The fourth was used as a nonirradiated control. From each aliquot, three samples were subjected to ESR spectroscopy. The sample was examined with a Bruker EMX 10/12 X-band spectrometer equipped with a cylindrical resonant cavity. The ESR spectrum was measured using recording parameters described in a previous report by our group (Mangiacotti et al. 2009). The mean signal intensity for each aliquot (the average of the values for the three samples) was used to investigate the dose-dependency of the ESR signal intensity, which is the dosimetric parameter. Its value was determined as the peak-peak height of the main component of the signal generated by the $\mathrm{CO}_{2}{ }^{-}$radicals normalized to the height of the marker signal and to the mass of the irradiated powder. The entire analytical procedure and 
the equipment used for measurements were subjected to rigorous quality controls. The identification of irradiated fish was carried out measuring the $g_{1}$ and $g_{2}$ factors of the acquired spectrum with the aid of spectrum-elaboration software and an appropriate marker kept in the cavity.

\section{Results}

Our tests confirmed that the method can be used on all three types of fish included in the program of intralaboratory validation (Table 1). In particular, we also analyzed the stability over time of the radio-induced signal, which is essential for the identification of an irradiated foodstuff at any time during its shelf-life. For each fish species tested, the results revealed evidence of signal fading at all dose levels used, although the percentage of fading decreased as the dose increased. In addition to the analytical performance of the proposed method, Table 1 also lists the relative radiosensitivities of the three species considered. These values represent the ratio of the slope of the regression curve obtained with the least squares method for a given species to the slope of the curve for gilthead sea breams, the species that proved to be the most sensitive. Given the characteristics described above, ESR spectroscopy is the method of choice for identifying irradiated fish if the test sample contains at least a few bone fragments.

\section{Discussion}

The results obtained in this study indicate that the proposed method is simple to use and reliable. For each fish species tested, samples of flesh and bone irradiated at doses ranging from 0.5 to $3 \mathrm{kGy}$ were correctly identified with a quantification limit (QL) of $0.5 \mathrm{kGy}$. Samples could be correctly identified up to 2 years after irradiation, a period that is longer than the shelf life of even frozen seafood products. In all three species, ESR signal intensity displayed linear correlation with the radiation dose used, with a minimum correlation coefficient $\left(\mathrm{R}^{2}\right)$ greater than 0.94 . The study of signal fading over a 2 -year period revealed substantial decreases in signal intensity in all samples. Maximum fading was observed in gilthead sea breams (up to $75 \%$ ). Swordfish was the species displaying the least amount of variation as a function of dose. These findings are in line with the results reported by other groups (Goulas et al. 2008). The results of a preliminary monitoring study conducted on a total of 25 randomly-collected samples of locally marketed fish ( 8 gilthead sea bream, 7 cod, 10 swordfish) revealed no instances of nonconformity with current labeling laws, and none of the fish sampled had been treated with ionizing radiation.

Table 1 Validation parameters: Quantification limit (QL), linearity, signal fading after 2 years, and relative sensitivity

\begin{tabular}{lllll}
\hline Fish & $\begin{array}{l}\text { Quantification } \\
\text { limit (kGy) }\end{array}$ & Linearity $\left(\mathrm{R}^{2}\right)$ & $\begin{array}{l}\text { Fading after } \\
\text { 2 years (\%) }\end{array}$ & $\begin{array}{l}\text { Relative } \\
\text { sensitivity }\end{array}$ \\
\hline Gilthead sea bream & 0.5 & 0.94 & $49-75 \%$ & 1 \\
Cod & 0.5 & 0.98 & $44-70 \%$ & 0.7 \\
Swordfish & 0.5 & 0.98 & $57-59 \%$ & 0.3 \\
\hline
\end{tabular}


Acknowledgements This study was carried out with the technical assistance of Mr. Donato Annicchiarico and Mr. Rosario Accettulli.

\section{References}

Goulas AE, Stahl M, Riganakos KA (2008) Effect of various parameters of irradiated fish and oregano using the ESR and PSL methods. Food Control 19:1076-1085

Mangiacotti M, Chiaravalle AE, Marchesani G, Pompa C (2009) Extension and validation of the UNI EN 1786 method for electron spin resonance (ESR) detection of irradiated turkey, pork and horse meat samples. Vet Res Comm 33(Suppl 1):237-239 